\title{
CELL CONTAMINATION AND BRANCHING PROCESSES IN A RANDOM ENVIRONMENT WITH IMMIGRATION
}

\author{
VINCENT BANSAYE, ${ }^{*}$ Université Pierre et Marie Curie et CNRS
}

\begin{abstract}
We consider a branching model for a population of dividing cells infected by parasites. Each cell receives parasites by inheritance from its mother cell and independent contamination from outside the cell population. Parasites multiply randomly inside the cell and are shared randomly between the two daughter cells when the cell divides. The law governing the number of parasites which contaminate a given cell depends only on whether the cell is already infected or not. We first determine the asymptotic behavior of branching processes in a random environment with state-dependent immigration, which gives the convergence in distribution of the number of parasites in a cell line. We then derive a law of large numbers for the asymptotic proportions of cells with a given number of parasites. The main tools are branching processes in a random environment and laws of large numbers for a Markov tree.
\end{abstract}

Keywords: Branching processes in a random environment with immigration; Markov chain indexed by a tree; empirical measure; renewal theorem

2000 Mathematics Subject Classification: Primary 60J80; 60J85; 60K37; 92C37; 92D25;

92D30

\section{Introduction}

We consider the following model for cell division with parasite infection and state-dependent contamination. The cell population starts from one single cell and divides in discrete time. At each generation,

(i) the parasites multiply randomly inside the cells,

(ii) each cell is contaminated by a random number of parasites which come from outside the cell population,

(iii) each cell divides into two daughter cells and the parasites are shared randomly between these two cells.

We will distinguish a first daughter cell from a second daughter cell by respectively labeling them 0 and 1 . We denote by $\mathbb{T}=\bigcup_{n \in \mathbb{N}}\{0,1\}^{n}$ the binary genealogical tree of the cell population, by $\mathbb{G}_{n}$ the set of cells in generation $n$, and by $Z_{i}$ the number of parasites of cell $i \in \mathbb{T}$. The labels $i 0$ and $i 1$ denote the two daughter cells of cell $i \in \mathbb{T}$.

In Section 1.1 we describe by a branching process the random multiplication and sharing of parasites in the cell, i.e. this branching process combines (i) and (iii). In Section 1.2 we

Received 23 May 2008; revision received 17 July 2009.

* Current address: CMAP, École Polytechnique, Route de Saclay, 91128 Palaiseau Cedex, France.

Email address: vincent.bansaye@polytechnique.edu 
describe the random contamination (ii) by immigration. Finally, in Section 1.3 we combine both in an independent and identically distributed (i.i.d.) manner to fully describe the model.

\subsection{Parasite infection and cell division}

For every cell, we randomly choose a mechanism for the multiplication of the parasites inside the cell and the sharing of parasites between the offspring of the cell when it divides. This mechanism is i.i.d. for every cell. Its distribution is specified by a random couple probability generating function (PGF) $\hat{f}$. This means that $\hat{f}$ is almost surely (a.s.) the PGF of a pair of random variables taking values in $\mathbb{N}$.

More precisely, let $\left(\hat{f}_{i}\right)_{i \in \mathbb{T}}$ be a sequence of i.i.d. PGFs distributed as $\hat{f}$. For each cell $i, \hat{f}_{i}$ gives the reproduction law and the sharing of the parasites' offspring in the following way. For every $i \in \mathbb{T}$, let $\left(X_{k}^{(0)}(i), X_{k}^{(1)}(i)\right)_{k \in \mathbb{N}}$ be a sequence of random variables such that, conditionally on $\hat{f}_{i}=\hat{g},\left(X_{k}^{(0)}(i), X_{k}^{(1)}(i)\right)_{k \in \mathbb{N}}$ are i.i.d. with common couple PGF $\hat{g}$ : for all $i \in \mathbb{T}, k \in \mathbb{N}$, and $s, t \in[0,1]$,

$$
\mathrm{E}\left(s^{X_{k}^{(0)}(i)} t^{X_{k}^{(1)}(i)} \mid \hat{f_{i}}=\hat{g}\right)=\hat{g}(s, t) .
$$

Then, in each generation, each parasite $k$ of the cell $i$ gives birth to $X_{k}^{(0)}(i)+X_{k}^{(1)}(i)$ children, $X_{k}^{(0)}(i)$ of which go into the first daughter cell and $X_{k}^{(1)}(i)$ of which go into the second daughter cell when the cell divides. This is a more general model for parasite infection and cell division than the model studied in [8], where there was no random environment ( $\hat{f}$ was deterministic) and the total number of parasites was given by a Galton-Watson process. See [15] for the original model in continuous time.

Our model also includes the following two natural models, with random binomial repartition of parasites. Let $Z$ be a random variable in $\mathbb{N}$, and let $\left(P_{i}\right)_{i \in \mathbb{T}}$ be an i.i.d. random variable in $[0,1]$. In each generation, every parasite multiplies independently with the same reproduction law $Z$. Thus, parasites follow a Galton-Watson process. Moreover, $P_{i}$ gives the mean fraction of parasites of the cell $i$ which goes into the first daughter cell when the cell divides. More precisely, conditionally on $P_{i}=p$, every parasite of the mother cell $i$ independently chooses the first daughter cell with probability $p$ (and the second daughter cell with probability $1-p$ ). It also contains the model that every parasite independently gives birth to a random cluster of parasites of size $Z$ and, conditionally on $P_{i}=p$, every cluster of parasite independently goes into the first cell with probability $p$ (and into the second cell with probability $1-p$ ).

We want to take into account the asymmetric repartition of parasites and so we do not make any assumption about $\hat{f}$. Indeed, unequal sharing has been observed when the cell divides; see, e.g. the experiments of Paepe, Paul and Taddei (see [20]), who infected the bacteria E. coli with a lysogen bacteriophage M13. In Section 6.1 we consider this model, where a cell receives parasites only by inheritance from its mother cell. We determine when the number of infected cells becomes negligible compared to the number of cells as the number of generations tends to $\infty$.

\subsection{State-dependent contamination}

In each generation, each cell may be contaminated by a random number of parasites, which also multiply randomly and are shared randomly between the two daughter cells. This contamination depends only on whether the cell already contains parasites or not.

More formally, if a cell $i$ contains $x$ parasites, the contamination brings $Y_{x}^{(0)}$ parasites to the first daughter cell of $i$ and $Y_{x}^{(1)}$ to the second daughter cell, where, for all $x \geq 1$,

$$
Y_{1}: \stackrel{\mathrm{D}}{=} Y_{x}^{(0)} \stackrel{\mathrm{D}}{=} Y_{x}^{(1)}, \quad Y_{0}: \stackrel{\mathrm{D}}{=} Y_{0}^{(0)} \stackrel{\mathrm{D}}{=} Y_{0}^{(1)} .
$$


Here, ' $\stackrel{D}{=}$ denotes equality in distribution. Moreover, we assume that contamination satisfies

$$
0<\mathrm{P}\left(Y_{0}=0\right)<1, \quad 0<\mathrm{P}\left(Y_{1}=0\right),
$$

which means that each noninfected cell may be contaminated with a positive probability, but the cells are not contaminated with probability 1.

This model contains the case where the contamination is independent of the number of parasites in the cell ( $Y_{0}$ and $Y_{1}$ are identically distributed). It also takes into account the case where only noninfected cells can be contaminated $\left(Y_{1}=0\right.$ a.s.), and the case where infected cells are 'weaker' and parasites contaminate them easier $\left(Y_{1} \geq Y_{0}\right.$ a.s.). For biological and technical reasons, we do not make $Y_{x}$ depend on $x \geq 1$. However, the results given here could be generalized to the case where the contamination depends on the number of parasites $x$ inside the cells as soon as $x$ is less than some fixed constant.

\subsection{Cell division with parasite infection and contamination}

We now describe the whole model (see also Figure 1). We start with a single cell with $k$ parasites and denote by $\mathrm{P}_{k}$ the associated probability. Unless stated otherwise, we assume that $k=0$.

For every cell $i \in \mathbb{T}$, conditionally on $Z_{i}=x$ and $\hat{f}_{i}=\hat{g}$, the numbers of parasites, $\left(Z_{i 0}, Z_{i 1}\right)$, of its two daughter cells are distributed as

$$
\sum_{k=1}^{x}\left(X_{k}^{(0)}(i), X_{k}^{(1)}(i)\right)+\left(Y_{x}^{(0)}(i), Y_{x}^{(1)}(i)\right),
$$

where

(i) $\left(X_{k}^{(0)}(i), X_{k}^{(1)}(i)\right)_{k \geq 1}$ is an i.i.d. sequence with common couple PGF $g$,

(ii) $\left(Y_{x}^{(0)}(i), Y_{x}^{(1)}(i)\right)$ is independent of $\left(X_{k}^{(0)}(i), X_{k}^{(1)}(i)\right)_{k \geq 1}$.

Moreover, $\left(\left(X_{k}^{(0)}(i), X_{k}^{(1)}(i)\right)_{k \geq 1},\left(Y_{x}^{(0)}(i), Y_{x}^{(1)}(i)\right)_{x \geq 0}\right)$ is i.i.d. for $i \in \mathbb{T}$.

This model is a Markov chain indexed by a tree. This subject has been studied in the literature (see, e.g. [5], [6], and [9]) in the symmetric independent case. That is, for all $(i, k) \in \mathbb{T} \times \mathbb{N}$,

$$
\mathrm{P}\left(\left(Z_{i 0}, Z_{i 1}\right)=\left(k_{0}, k_{1}\right) \mid Z_{i}=k\right)=\mathrm{P}\left(Z_{i 0}=k_{0} \mid Z_{i}=k\right) \mathrm{P}\left(Z_{i 0}=k_{1} \mid Z_{i}=k\right) .
$$

But this identity does not hold here since we are interested in unequal sharing of parasites. Guyon [12] proved limit theorems for a Markov chain indexed by a binary tree where asymmetry and dependence are allowed. His theorem is the key argument used here to prove the convergence of asymptotic proportions of cells with a given number of parasites. Indeed, contamination ensures that the process which counts the number of parasites along the random walk on the binary tree of the cell population is ergodic and nontrivial (see Section 5). This ergodic property is the fundamental hypothesis of Guyon's law of large numbers result. Let us now introduce more precisely the process which gives the number of parasites in a random cell line.

Let $\left(a_{j}\right)_{j \in \mathbb{N}}$ be an i.i.d. sequence independent of $\left(Z_{i}\right)_{i \in \mathbb{T}}$ such that

$$
\mathrm{P}\left(a_{1}=0\right)=\mathrm{P}\left(a_{1}=1\right)=\frac{1}{2} .
$$

Denote by $f^{(0)}$ and $f^{(1)}$ the random PGFs which give the laws of the size of the offspring of a parasite which go into the first and second daughter cells, respectively:

$$
f^{(0)}(s):=\hat{f}(s, 1) \quad \text { a.s., } \quad f^{(1)}(t):=\hat{f}(1, t) \quad \text { a.s., } \quad s, t \in[0,1] .
$$


Generation 0

Generation 1

Generation 2

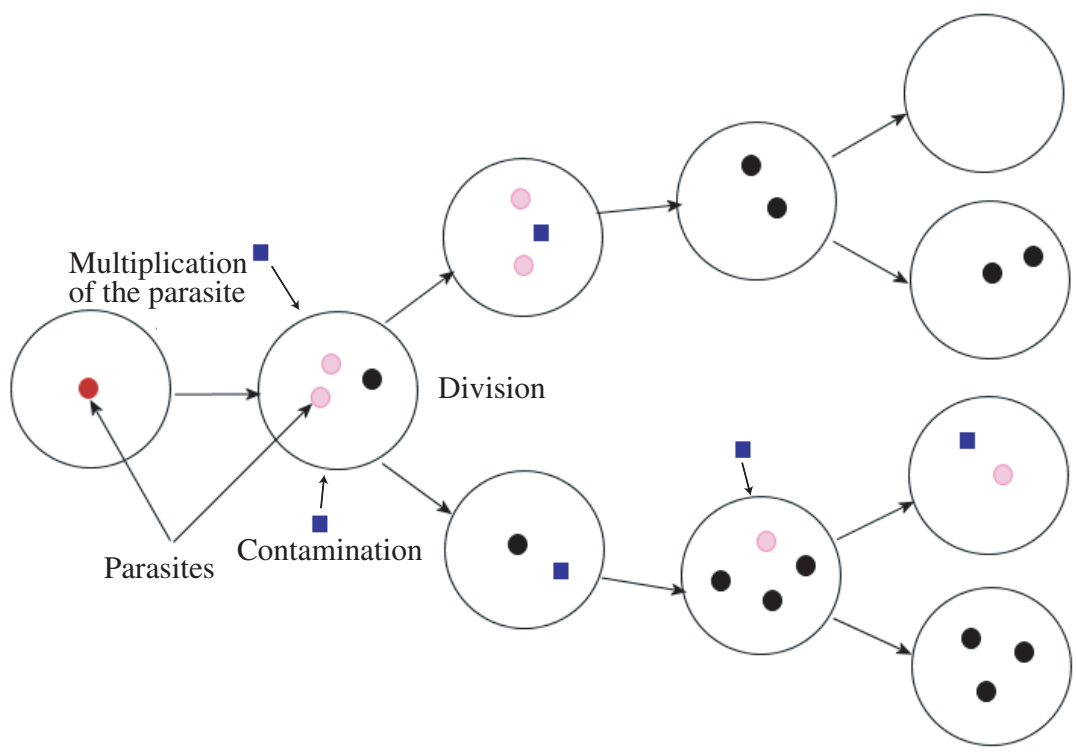

FIGURE 1: Cell division with multiplication of parasites, random sharing, and contamination. Each parasite gives birth to a random number of light parasites and dark parasites. Light parasites go into the first daughter cell, dark parasites go into the second daughter cell, and square parasites contaminate the cells from outside the cell population. But light, dark, and square parasites then behave in the same way.

Let $f$ be the mixed generating function of $f^{(0)}$ and $f^{(1)}$, i.e.

$$
\mathrm{P}(f \in \mathrm{d} g)=\frac{\mathrm{P}\left(f^{(0)} \in \mathrm{d} g\right)+\mathrm{P}\left(f^{(1)} \in \mathrm{d} g\right)}{2} .
$$

Then $\left(Z_{n}\right)_{n \in \mathbb{N}}=\left(Z_{\left(a_{1}, a_{2}, \ldots, a_{n}\right)}\right)_{n \in \mathbb{N}}$ is a branching process in a random environment with immigration that depends on whether the state is zero or not: the reproduction law is given by its $\operatorname{PGF} f$, the immigration law in zero is distributed as $Y_{0}$, and the immigration law in $k \geq 1$ is distributed as $Y_{1}$. Thus, we first need to prove asymptotic results for this process.

\section{Main results}

Galton-Watson processes with immigration are well known (see, e.g. [1], [4], and [17]). If the process is subcritical and the expectation of the logarithm of the immigration is finite, then it converges in distribution to a finite random variable. Otherwise, it tends to $\infty$ in probability. Key [13] obtained the analogous result for branching processes in a random environment with immigration (BPREI), in the subcritical case, with finite expectation of the logarithm. Actually, he stated results for multitype BPREI, which have been complemented by Roitershtein [18], who obtained a strong law of large numbers and a central limit theorem for the partial sum.

In Section 4 we give the asymptotic behavior of BPREI in the critical and supercritical cases, and the case where the expectation of the logarithm of the immigration is infinite. To obtain these 
results, we use some general statements on Markov processes (Section 3.2), classical arguments for Galton-Watson processes with immigration (see [17], which was inspired by [1]), and the tail of the time when a branching process in a random environment with immigration returns to 0 in the subcritical case, which was proved in [13].

We can then state results about branching processes in a random environment, $\left(Z_{n}\right)_{n \in \mathbb{N}}$, with immigration that depends on whether the state is zero or not (Section 5) using coupling arguments and the results of Section 3.2. This process gives the number of parasites along a random cell line. Recalling that immigration in state zero is distributed as $Y_{0}$ and immigration in state $k \geq 1$ is distributed as $Y_{1}$, we prove the following expected result.

Theorem 1. (i) If $\mathrm{E}\left(\log \left(f^{\prime}(1)\right)\right)<0$ and $\max \left(\mathrm{E}\left(\log ^{+}\left(Y_{i}\right)\right): i=0,1\right)<\infty$, then there exists a finite random variable $Z_{\infty}$ such that, for every $k \in \mathbb{N}, Z_{n}$ starting from $k$ converges in distribution to $Z_{\infty}$ as $n \rightarrow \infty$.

(ii) If $\mathrm{E}\left(\log \left(f^{\prime}(1)\right)\right) \geq 0$ or $\max \left(\mathrm{E}\left(\log ^{+}\left(Y_{i}\right)\right): i=0,1\right)=\infty$, then $Z_{n}$ converges in probability to $\infty$ as $n \rightarrow \infty$.

With additional assumptions, in Section 5 we provide an estimate of the rate of convergence of $\left(Z_{n}\right)_{n \in \mathbb{N}}$ depending on the initial state. Then, in Section 6 , we prove asymptotic results on the population of cells in generation $n$ as $n \rightarrow \infty$.

Firstly, we consider the case when there is no contamination, i.e. $Y_{0}=Y_{1}=0$ a.s. We determine when the organism recovers, that is, when the number of infected cells becomes negligible compared to the total number of cells. As stated in Proposition 2, below, the recovery occurs a.s. if and only if $\mathrm{E}\left(\log \left(f^{\prime}(1)\right)\right) \leq 0$. Thus, we generalize the results of Section 3 of [8] to a random environment. Again, for any reproduction rate of parasites, we can find a necessary and sufficient condition on the sharing of their offspring so that the organism recovers a.s.

As explained in the introduction, a natural example is the random binomial repartition of parasites. If the reproduction of parasites is given by the random variable $Z$, and the random parameter of the binomial repartition is given by $P \in[0,1]$, the almost-sure recovery criterion becomes

$$
\log (\mathrm{E}(Z)) \leq \mathrm{E}\left(\log \left(\frac{1}{P}\right)\right) .
$$

Secondly, we take into account the contamination by parasites from outside the cell population, assuming that (1) holds. We focus on proportions of cells in generation $n$ with a given number of parasites:

$$
F_{k}(n):=\frac{\#\left\{i \in \mathbb{G}_{n}: Z_{i}=k\right\}}{2^{n}}, \quad k \in \mathbb{N}
$$

Using [12] and Theorem 1, we prove the following law of large numbers.

Theorem 2. If $\mathrm{E}\left(\log \left(f^{\prime}(1)\right)\right)<0$ and $\max \left(\mathrm{E}\left(\log ^{+}\left(Y_{i}\right)\right): i=0,1\right)<\infty$, then, for every $k \in \mathbb{N}, F_{k}(n)$ converges in probability to a deterministic number $f_{k}$ as $n \rightarrow \infty$, such that $f_{0}>0$ and $\sum_{k=0}^{\infty} f_{k}=1$. Otherwise, for every $k \in \mathbb{N}, F_{k}(n)$ converges in probability to 0 as $n \rightarrow \infty$.

Finally, in Section 7 we give the asymptotic behavior of the total number of parasites in generation $n$ in the case where the growth of parasites follows a Galton-Watson process and the contamination does not depend on the state of the cell. 


\section{Preliminaries}

We first recall some results about branching processes in a random environment (BPRE) and then about Markov chains, both of which will be useful to study BPREI, $\left(Z_{n}\right)_{n \in \mathbb{N}}$. Recall that we denote by $k$ the initial number of parasites and by $\mathrm{P}_{k}$ the associated probability.

\subsection{Branching processes in a random environment}

We consider here BPRE, $\left(Z_{n}\right)_{n \in \mathbb{N}}$, specified by a sequence of i.i.d. generating functions $\left(f_{n}\right)_{n \in \mathbb{N}}$ distributed as $f$ [2], [3], [19]. More precisely, conditionally on the environment $\left(f_{n}\right)_{n \in \mathbb{N}}$, particles in generation $n$ reproduce independently of each other and their offspring has generating function $f_{n}$. Then $Z_{n}$ is the number of particles in generation $n$ and $Z_{n+1}$ is the sum of $Z_{n}$ independent random variables with generating function $f_{n}$. That is, for every $n \in \mathbb{N}$,

$$
\mathrm{E}\left(s^{Z_{n+1}} \mid Z_{0}, \ldots, Z_{n} ; f_{0}, \ldots, f_{n}\right)=f_{n}(s)^{Z_{n}}, \quad 0 \leq s \leq 1 .
$$

Thus, denoting by $F_{n}:=f_{0} \circ \cdots \circ f_{n-1}$, we have, for every $k \in \mathbb{N}$,

$$
\mathrm{E}_{k}\left(s^{Z_{n+1}} \mid f_{0}, \ldots, f_{n}\right)=\mathrm{E}\left(s^{Z_{n+1}} \mid Z_{0}=k, f_{0}, \ldots, f_{n}\right)=F_{n}(s)^{k}, \quad 0 \leq s \leq 1 .
$$

When the environments are deterministic (i.e. $f$ is a deterministic generating function), this process is the Galton-Watson process with reproduction law $N$, where $f$ is the generating function of $N$.

The process $\left(Z_{n}\right)_{n \in \mathbb{N}}$ is called subcritical, critical, or supercritical if

$$
\mathrm{E}\left(\log \left(f^{\prime}(1)\right)\right)
$$

is negative, zero, or positive, respectively. This process becomes extinct a.s.:

$$
\mathrm{P}\left(\text { there exists } n \in \mathbb{N}: Z_{n}=0\right)=1,
$$

if and only if it is subcritical or critical [3] (see [11] for finer results).

In the critical case we make the following integrability assumption:

$$
0<\mathrm{E}\left(\log \left(f_{0}^{\prime}(1)\right)^{2}\right)<\infty, \quad \mathrm{E}\left(\frac{\left[1+\log \left(f_{0}^{\prime}(1)\right)\right] f_{0}^{\prime \prime}(1)}{2 f_{0}^{\prime}(1)}\right)<\infty,
$$

so that there exist $0<c_{1}<c_{2}<\infty$ such that, for every $n \in \mathbb{N}$ (see [16]),

$$
\frac{c_{1}}{\sqrt{n}} \leq \mathrm{P}\left(Z_{n}>0\right) \leq \frac{c_{2}}{\sqrt{n}} .
$$

See [7] for more general results in the critical case.

\subsection{Markov chains}

We now consider a Markov chain $\left(Z_{n}\right)_{n \in \mathbb{N}}$ taking values in $\mathbb{N}$ and introduce the first time $T_{0}$ when $\left(Z_{n}\right)_{n \in \mathbb{N}}$ visits 0 after time 0 :

$$
T_{0}:=\inf \left\{j>0: Z_{j}=0\right\} .
$$

Define

$$
u_{n}:=\mathrm{P}_{0}\left(Z_{n}=0\right), \quad u_{\infty}:=\frac{1}{\mathrm{E}_{0}\left(T_{0}\right)}
$$


(with the convention that $1 / \infty=0)$. We assume that $0<\mathrm{P}_{0}\left(Z_{1}=0\right)<1$, and we give the asymptotic behavior of $\left(Z_{n}\right)_{n \in \mathbb{N}}$. The first part of Lemma 1(i),below, is the classical ergodic property for an aperiodic positive recurrent Markov chain, and we provide an estimate of the speed of convergence depending on the initial state. Then Lemma 1(ii) gives the null recurrent case, which is also a classical result.

Lemma 1. (i) If, for every $k \in \mathbb{N}, \mathrm{E}_{k}\left(T_{0}\right)<\infty$ then $Z_{n}$ starting from $k$ converges in distribution to a finite random variable $Z_{\infty}$, which does not depend on $k$ and verifies

$$
\mathrm{P}\left(Z_{\infty}=0\right)>0 .
$$

Moreover, there exists $A>0$ such that, for all $n, k \in \mathbb{N}$,

$$
\begin{aligned}
& \sum_{l \in \mathbb{N}}\left|\mathrm{P}_{k}\left(Z_{n}=l\right)-\mathrm{P}\left(Z_{\infty}=l\right)\right| \\
& \quad \leq A\left[\sup _{n / 2 \leq l \leq n}\left\{\left|u_{l}-u_{\infty}\right|\right\}+\mathrm{E}_{0}\left(T_{0} \mathbf{1}_{\left\{T_{0}>n / 4\right\}}\right)+\mathrm{E}_{k}\left(T_{0} \mathbf{1}_{\left\{T_{0}>n / 4\right\}}\right)\right] .
\end{aligned}
$$

(ii) If $\mathrm{E}_{0}\left(T_{0}\right)=\infty$ and, for every $l \in \mathbb{N}, \mathrm{P}_{l}\left(T_{0}<\infty\right)>0$, then, for every $k \in \mathbb{N}, Z_{n} \rightarrow \infty$ in $\mathrm{P}_{k}$-probability as $n \rightarrow \infty$.

Proof. (i) First, note that, by the Markov property, for every $n \in \mathbb{N}$,

$$
\begin{aligned}
\left|\mathrm{P}_{k}\left(Z_{n}=0\right)-u_{\infty}\right| & =\left|\sum_{j=1}^{n} \mathrm{P}_{k}\left(T_{0}=j\right) \mathrm{P}_{0}\left(Z_{n-j}=0\right)-u_{\infty}\right| \\
& \leq \sum_{j=1}^{n} \mathrm{P}_{k}\left(T_{0}=j\right)\left|u_{n-j}-u_{\infty}\right|+u_{\infty} \mathrm{P}_{k}\left(T_{0}>n\right) .
\end{aligned}
$$

On the event $\left\{T_{0} \leq n\right\}$, define $R_{n}$ as the last passage time of $\left(Z_{n}\right)_{n \in \mathbb{N}}$ by 0 before time $n$ :

$$
R_{n}:=\sup \left\{j \leq n: Z_{j}=0\right\} .
$$

For $l \in \mathbb{N}$, by the Markov property,

$$
\begin{aligned}
a \mathrm{P}_{k}\left(Z_{n}=l\right) & =\mathrm{P}_{k}\left(T_{0}>n, Z_{n}=l\right)+\sum_{i=0}^{n} \mathrm{P}_{k}\left(T_{0} \leq n, R_{n}=n-i, Z_{n}=l\right) \\
& =\mathrm{P}_{k}\left(T_{0}>n, Z_{n}=l\right)+\sum_{i=0}^{n} \mathrm{P}_{k}\left(Z_{n-i}=0\right) \mathrm{P}_{0}\left(Z_{i}=l, T_{0}>i\right) .
\end{aligned}
$$

Now define

$$
\alpha_{l}:=u_{\infty} \sum_{i=0}^{\infty} \mathrm{P}_{0}\left(Z_{i}=l, T_{0}>i\right)
$$

We then have

$$
\begin{aligned}
\left|\mathrm{P}_{k}\left(Z_{n}=l\right)-\alpha_{l}\right| \leq & \mathrm{P}_{k}\left(T_{0}>n, Z_{n}=l\right)+u_{\infty} \sum_{i=n+1}^{\infty} \mathrm{P}\left(Z_{i}=l, T_{0}>i\right) \\
& +\sum_{i=0}^{n} \mathrm{P}\left(Z_{i}=l, T_{0}>i\right)\left|u_{\infty}-\mathrm{P}_{k}\left(Z_{n-i}=0\right)\right| .
\end{aligned}
$$


Summing over $l$ leads to

$$
\begin{aligned}
\sum_{l \in \mathbb{N}}\left|\mathrm{P}_{k}\left(Z_{n}=l\right)-\alpha_{l}\right| \leq & \mathrm{P}_{k}\left(T_{0}>n\right)+u_{\infty} \mathrm{E}_{0}\left(T_{0} \mathbf{1}_{\left\{T_{0}>n+1\right\}}\right) \\
& +\sum_{i=0}^{n} \mathrm{P}\left(T_{0}>i\right)\left|u_{\infty}-\mathrm{P}_{k}\left(Z_{n-i}=0\right)\right| .
\end{aligned}
$$

Moreover, using (3), we have, for all $0 \leq n_{0} \leq n$,

$$
\begin{aligned}
& \sum_{i=0}^{n} \mathrm{P}\left(T_{0}>i\right)\left|u_{\infty}-\mathrm{P}_{k}\left(Z_{n-i}=0\right)\right| \\
& \quad \leq \sum_{i=0}^{n} \mathrm{P}_{0}\left(T_{0}>i\right)\left(\sum_{j=1}^{n-i} \mathrm{P}_{k}\left(T_{0}=j\right)\left|u_{n-i-j}-u_{\infty}\right|+u_{\infty} \mathrm{P}_{k}\left(T_{0}>n-i\right)\right) \\
& \quad \leq \sum_{i=0}^{n} \mathrm{P}_{0}\left(T_{0}>i\right) \sum_{j=1}^{n-i} \mathrm{P}_{k}\left(T_{0}=j\right)\left|u_{n-i-j}-u_{\infty}\right|+u_{\infty} \sum_{i=0}^{n} \mathrm{P}_{0}\left(T_{0}>i\right) \mathrm{P}_{k}\left(T_{0}>n-i\right) .
\end{aligned}
$$

Finally, denoting by $M:=\sup _{n \in \mathbb{N}}\left\{\left|u_{n}-u_{\infty}\right|\right\}$,

$$
\begin{aligned}
& \sum_{i=0}^{n} \mathrm{P}_{0}\left(T_{0}>i\right) \sum_{j=1}^{n-i} \mathrm{P}_{k}\left(T_{0}=j\right)\left|u_{n-i-j}-u_{\infty}\right| \\
& \leq \sup _{n_{0} \leq l \leq n}\left\{\left|u_{l}-u_{\infty}\right|\right\} \sum_{i=0}^{n} \mathrm{P}_{0}\left(T_{0}>i\right) \sum_{j=1}^{n-i} \mathrm{P}_{k}\left(T_{0}=j\right) \mathbf{1}_{\left\{n-i-j \geq n_{0}\right\}} \\
& \quad+M \sum_{i=0}^{n} \mathrm{P}_{0}\left(T_{0}>i\right) \sum_{j=1}^{n-i} \mathrm{P}_{k}\left(T_{0}=j\right) \mathbf{1}_{\left\{n-i-j<n_{0}\right\}} \\
& \leq \sup _{n_{0} \leq l \leq n}\left\{\left|u_{l}-u_{\infty}\right|\right\} \sum_{i=0}^{n} \mathrm{P}_{0}\left(T_{0}>i\right) \sum_{j=1}^{n-i} \mathrm{P}_{k}\left(T_{0}=j\right) \\
& \quad+M \sum_{i=0}^{n-n_{0}} \mathrm{P}_{0}\left(T_{0}>i\right) \mathrm{P}_{k}\left(T_{0}>n-n_{0}-i\right) .
\end{aligned}
$$

Combining (4), (5), and (6), and using the facts that

$$
\begin{gathered}
\sum_{i=0}^{n} \mathrm{P}_{0}\left(T_{0}>i\right) \mathrm{P}_{k}\left(T_{0}>n-i\right) \leq \mathrm{E}_{0}\left(T_{0} \mathbf{1}_{\left\{T_{0}>n / 2\right\}}\right)+\mathrm{E}_{k}\left(T_{0} \mathbf{1}_{\left\{T_{0}>n / 2\right\}}\right), \\
\sum_{i=0}^{n-n_{0}} \mathrm{P}_{0}\left(T_{0}>i\right) \mathrm{P}_{k}\left(T_{0} \geq n-n_{0}-i\right) \leq \mathrm{E}_{0}\left(T_{0} \mathbf{1}_{\left\{T_{0}>\left(n-n_{0}\right) / 2\right\}}\right)+\mathrm{E}_{k}\left(T_{0} \mathbf{1}_{\left.\left\{T_{0}>n-n_{0}\right) / 2\right\}}\right),
\end{gathered}
$$

we obtain, for all $0 \leq n_{0} \leq n$,

$$
\begin{aligned}
\sum_{l \in \mathbb{N}}\left|\mathrm{P}_{k}\left(Z_{n}=l\right)-\alpha_{l}\right| \leq & \mathrm{P}_{k}\left(T_{0}>n\right)+u_{\infty} \mathrm{E}_{0}\left(T_{0} \mathbf{1}_{\left\{T_{0}>n+1\right\}}\right)+\sup _{n_{0} \leq l \leq n}\left\{\left|u_{l}-u_{\infty}\right|\right\} \mathrm{E}_{0}\left(T_{0}\right) \\
& +\left[u_{\infty}+M\right]\left[\mathrm{E}_{0}\left(T_{0} \mathbf{1}_{\left\{T_{0}>\left(n-n_{0}\right) / 2\right\}}\right)+\mathrm{E}_{k}\left(T_{0} \mathbf{1}_{\left\{T_{0}>\left(n-n_{0}\right) / 2\right\}}\right)\right] .
\end{aligned}
$$


As $\mathrm{P}_{0}\left(Z_{1}=0\right)>0$, by the renewal theorem [10], $u_{n} \rightarrow u_{\infty}$ as $n \rightarrow \infty$. Adding that $\mathrm{E}_{k}\left(T_{0}\right)<\infty$ and $\mathrm{E}_{0}\left(T_{0}\right)<\infty$ ensures that

$$
\sum_{l \in \mathbb{N}}\left|\mathrm{P}_{k}\left(Z_{n}=l\right)-\alpha_{l}\right| \rightarrow 0 \quad \text { as } n \rightarrow \infty,
$$

which proves that $Z_{n}$ starting from $k$ converges in distribution to a random variable $Z_{\infty}$ that does not depend on $k$.

The inequality of (i) is obtained by letting $n_{0}=n / 2$ in (7).

(ii) If $\mathrm{E}_{0}\left(T_{0}\right)=\infty$ then, again by the renewal theorem [10],

$$
u_{n} \rightarrow 0 \quad \text { as } n \rightarrow \infty
$$

So

$$
D_{n}=\inf \left\{k-n: k \geq n, Z_{k}=0\right\} \rightarrow \infty \text { in probability, as } n \rightarrow \infty .
$$

Assume that there exist $l \in \mathbb{N}, \varepsilon>0$, and an increasing sequence of integers $\left(u_{n}\right)_{n \in \mathbb{N}}$ such that

$$
\mathrm{P}_{k}\left(Z_{u_{n}}=l\right) \geq \varepsilon
$$

As $\mathrm{P}_{l}\left(T_{0}<\infty\right)>0$ by hypothesis, there exists $N>0$ such that

$$
\mathrm{P}_{l}\left(T_{0}=N\right)>0 .
$$

Thus, by the Markov property,

$$
\mathrm{P}_{k}\left(Z_{u_{n}+K}=0\right) \geq \mathrm{P}_{k}\left(Z_{u_{n}}=l\right) \mathrm{P}_{l}\left(T_{0}=N\right) \geq \varepsilon \mathrm{P}_{l}\left(T_{0}=N\right) .
$$

Then, for all $n \in \mathbb{N}$,

$$
\mathrm{P}_{k}\left(D_{u_{n}} \leq N\right) \geq \varepsilon \mathrm{P}_{l}\left(T_{0}=N\right)>0,
$$

which is in contradiction with the fact that $D_{n} \rightarrow \infty$ in $\mathrm{P}_{k}$ as $n \rightarrow \infty$. Then, $\mathrm{P}_{k}\left(Z_{n}=l\right) \rightarrow 0$ as $n \rightarrow \infty$.

\section{Branching processes in a random environment with immigration}

We consider here BPRE, $\left(Z_{n}\right)_{n \in \mathbb{N}}$, whose reproduction law is given by the random PGF $f$, and we add at each $(n+1)$ th generation a random number of immigrants, $Y_{n}$, i.i.d. as a random variable $Y$ such that

$$
\mathrm{P}(Y=0)>0 .
$$

More precisely, for every $n \in \mathbb{N}$,

$$
Z_{n+1}=Y_{n}+\sum_{j=1}^{Z_{n}} X_{j}
$$

where $\left(X_{j}\right)_{j \in \mathbb{N}}, Y_{n}$, and $Z_{n}$ are independent and, conditionally on $f_{n}=g$, the $\left(X_{j}\right)_{j \in \mathbb{N}}$ are i.i.d. with common PGF $g$.

Note that if the contamination is not dependent on whether the cell is already infected or not (i.e. $Y_{0}$ and $Y_{1}$ are identically distributed), then the number of parasites in a random cell line defined in the introduction is a branching process in a random environment with immigration, whose reproduction law is given by $f$ and whose immigration is given by $Y \stackrel{\mathrm{D}}{=} Y_{0} \stackrel{\mathrm{D}}{=} Y_{1}$. 
We now give the asymptotic behavior of this process. These results are classical for the Galton-Watson process with immigration [1], [17]. We follow the same method in the case of a random environment for the subcritical and supercritical cases. In Proposition (ii), below, we use the tail of the time,

$$
T_{0}:=\inf \left\{n>0: Z_{n}=0\right\},
$$

when the process returns to 0 in the subcritical case, which was proved in [13], and we use the results of Section 3.2 in the critical case.

Proposition 1. (i) If $\mathrm{E}\left(\log \left(f^{\prime}(1)\right)\right)<0$ and $\mathrm{E}\left(\log ^{+}(Y)\right)<\infty$, then $Z_{n}$ converges in distribution to a finite random variable as $n \rightarrow \infty$ and $\lim _{n \rightarrow \infty} \mathrm{P}\left(Z_{n}=0\right)>0$. Otherwise, $Z_{n} \rightarrow \infty$ as $n \rightarrow \infty$.

(ii) If $\mathrm{E}\left(\log \left(f^{\prime}(1)\right)\right)<0$ and there exists $q>0$ such that $\mathrm{E}\left(Y^{q}\right)<\infty$, then there exist $c, d>0$ such that, for every $n \in \mathbb{N}$,

$$
\mathrm{P}\left(T_{0}>n\right) \leq c \mathrm{e}^{-d n} .
$$

(iii) Assume that $\mathrm{E}\left(f^{\prime}(1)^{-1}\right)<1$ and $\mathrm{E}\left(\log ^{+}(Y)\right)<\infty$. Then there exists a finite random variable $W$ such that

$$
\left[\prod_{j=0}^{n-1} f_{j}^{\prime}(1)\right]^{-1} Z_{n} \rightarrow W \quad \text { in } \mathrm{P} \text {, as } n \rightarrow \infty
$$

Note also that, by the Borel-Cantelli lemma, if $\mathrm{E}\left(\log ^{+}\left(Y_{1}\right)\right)=\infty$ then, for every $c>1$,

$$
\limsup _{n \rightarrow \infty} c^{-n} Z_{n}=\infty \quad \text { a.s., }
$$

since $Z_{n} \geq Y_{n}$ a.s. Moreover, the proof of Theorem 3 in Section 5 provides another approach to prove that $\left(Z_{n}\right)_{n \in \mathbb{N}}$ tends to $\infty$ if $\mathrm{E}\left(\log ^{+}(Y)\right)=\infty$.

Proof of Proposition 1(i) and (ii) in the subcritical case: $\mathrm{E}\left(\log \left(f^{\prime}(1)\right)\right)<0$. The subcritical case with the assumption that $\mathrm{E}\left(\log ^{+}(Y)\right)<\infty$ is handled in [13]: the first part of (i) is [13, Theorem 3.3] and (ii) is a consequence of [13, Theorem 4.2].

We now focus on the $\mathrm{E}\left(\log ^{+}(Y)\right)=\infty$ case and prove that $Z_{n}$ converges in probability to $\infty$. The proof is similar to the Galton-Watson case (see [1] or [17]). First, by the Borel-Cantelli lemma,

$$
\limsup _{k \rightarrow \infty} \frac{\log ^{+}\left(Y_{k}\right)}{k}=\infty \quad \text { a.s. }
$$

Then, for every $c \in(0,1)$,

$$
\limsup _{k \rightarrow \infty} c^{k} Y_{k}=\infty \quad \text { a.s. }
$$

Note that

$$
Z_{n}=\sum_{k=0}^{n-1} Z_{k, n}
$$

where $Z_{k, n}$ is the number of descendants in the $n$th generation of immigrants in the $(n-k)$ th generation. Thus, denoting by $Y_{k, n}$ the number of immigrants in the $(n-k)$ th generation and 
by $X_{j}(k, n)$ the number of descendants in the $n$th generation of immigrant $j$ in the $(n-k)$ th generation, we have

$$
Z_{n}=\sum_{k=0}^{n-1} \sum_{j=1}^{Y_{k, n}} X_{j}(k, n) .
$$

This sum increases stochastically as $n$ tends to $\infty$ and converges in distribution to

$$
Z_{\infty}=\sum_{k=0}^{\infty} \sum_{j=1}^{Y_{k}} X_{j}(k),
$$

where, conditionally on $\left(f_{j}: j \in \mathbb{N}\right)$, the $\left(X_{j}(k): j \in \mathbb{N}, k \in \mathbb{N}\right)$ are independent and the PGF of $X_{j}(k)$ is equal to $f_{k-1} \circ \cdots \circ f_{0}$. Roughly speaking, $X_{j}(k)$ is the contribution of immigrant $j$, which arrives $k$ generations before the 'final time' $\infty$. The integer $X_{j}(k)$ is the population in generation $k$ of a branching process in a random environment without immigration starting from 1 .

Assume now that $Z_{\infty}<\infty$ with a positive probability. As the $\left(X_{j}(k): k \in \mathbb{N}, 1 \leq j \leq Y_{k}\right)$ are integers, then, conditionally on $Z_{\infty}<\infty$, only a finite number of them are positive. Thus, by the Borel-Cantelli lemma, conditionally on $\left(Z_{\infty}<\infty, Y_{k}: k \in \mathbb{N}, f_{j}: j \in \mathbb{N}\right)$,

$$
\sum_{k=0}^{\infty} Y_{k} \mathrm{P}\left(X_{1}(k)>0\right)<\infty \text { a.s. }
$$

Moreover, by convexity, for all PGFs $g$ and $s \in[0,1]$,

$$
\frac{1-g(s)}{1-s}=\frac{g(1)-g(s)}{1-s} \geq \frac{g(1)-g(0)}{1-0}=1-g(0), \quad 0 \leq s \leq 1 .
$$

Then $1-g(s) \geq(1-g(0))(1-s)$ and, by induction, we have, for every $k \in \mathbb{N}$,

$$
\begin{aligned}
\mathrm{P}\left(X_{1}(k)>0 \mid f_{j}: j \in \mathbb{N}\right) & =1-f_{k-1} \circ \cdots \circ f_{0}(0) \\
& \geq \prod_{j=0}^{k-1}\left(1-f_{j}(0)\right) \\
& =\exp \left(S_{k}\right),
\end{aligned}
$$

where $S_{k}:=\sum_{j=0}^{k-1} \log \left(1-f_{j}(0)\right)$. Thus, conditionally on $\left(Z_{\infty}<\infty, Y_{k}: k \in \mathbb{N}, f_{j}: j \in\right.$ $\mathbb{N})$,

$$
\sum_{k=0}^{\infty} Y_{k} \exp \left(S_{k}\right)<\infty \quad \text { a.s. }
$$

Thus, on the event $\left\{Z_{\infty}<\infty\right\}$, which has a positive probability, we obtain

$$
\sum_{k=0}^{\infty} Y_{k} \exp \left(S_{k}\right)<\infty \text { a.s. }
$$

Moreover, $S_{n}$ is a random walk with negative drift $\mathrm{E}\left(\log \left(1-f_{0}(0)\right)\right)$. So letting $\alpha<\mathrm{E}(\log (1-$ $\left.\left.f_{0}(1)\right)\right), \mathrm{P}\left(S_{n}<\alpha n\right)$ decreases exponentially by classical large deviation results. Then, by the Borel-Cantelli lemma, $S_{n}$ is less than $\alpha n$ for a finite number of $n$, and

$$
L:=\inf _{n \in \mathbb{N}}\left\{S_{n}-\alpha n\right\}>-\infty \text { a.s. }
$$


Using the fact that, for every $k \in \mathbb{N}, S_{k} \geq \alpha k+L$ a.s., we obtain

$$
\sum_{k=0}^{\infty} \exp (\alpha k) Y_{k}<\infty,
$$

with positive probability. This is in contradiction with (9). Then $Z_{\infty}=\infty$ a.s. and $Z_{n}$ converges in probability to $\infty$ as $n \rightarrow \infty$.

Proof of Proposition 1(i) in the critical and supercritical cases: $\mathrm{E}\left(\log \left(f^{\prime}(1)\right)\right) \geq 0$. First, we focus on the critical case. Recall that $T_{0}=\inf \left\{j>0: Z_{j}=0\right\}$, and consider $\left(\bar{Z}_{n}\right)_{n \in \mathbb{N}}$ to be the BPRE associated with $\left(Z_{n}\right)_{n \in \mathbb{N}}$, that is, the critical BPRE with reproduction law $f$ and no immigration. Thanks to (2), there exists $c_{1}>0$ such that, for every $n \in \mathbb{N}$,

$$
\mathrm{P}_{1}\left(\bar{Z}_{n}>0\right) \geq \frac{c_{1}}{\sqrt{n}} .
$$

Adding that

$$
\mathrm{P}_{1}\left(T_{0}>n\right)=\mathrm{P}_{1}\left(Z_{n}>0\right) \geq \mathrm{P}_{1}\left(\bar{Z}_{n}>0\right)
$$

ensures that

$$
\mathrm{E}_{1}\left(T_{0}\right)=\infty .
$$

Then $\mathrm{E}_{0}\left(T_{0}\right)=\infty$, since the BPREI, $\left(Z_{n}\right)_{n \in \mathbb{N}}$, starting from 1 are stochastically larger than $\left(Z_{n}\right)_{n \in \mathbb{N}}$ starting from 0 . Moreover, for all $k \in \mathbb{N}, \mathrm{P}_{k}\left(T_{0}<\infty\right)>0$, since $\mathrm{P}_{k}\left(\bar{T}_{0}<\infty\right)=1$ and $\mathrm{P}(Y=0)>0$. Then Lemma 1(ii) ensures that $Z_{n} \rightarrow \infty$ in $\mathrm{P}$ as $n \rightarrow \infty$.

For the supercritical case, follow the proof in the critical case (or use the result with a coupling argument) to show that $Z_{n} \rightarrow \infty$ in probability as $n \rightarrow \infty$.

Proof of Proposition 1(iii). Again, we follow the proof in [17]. If $\mathrm{E}\left(\log ^{+}(Y)\right)<\infty$, by the Borel-Cantelli lemma,

$$
\limsup _{k \rightarrow \infty} \frac{\log ^{+}\left(Y_{k}\right)}{k}=0 .
$$

Then, for every $c>1$,

$$
\sum_{k=0}^{\infty} c^{-k} Y_{k}<\infty \text { a.s. }
$$

Define

$$
P_{n}:=\left[\prod_{j=0}^{n-1} f_{j}^{\prime}(1)\right]^{-1},
$$

and denote by $\mathcal{F}_{n}$ the $\sigma$-field generated by $\left(Z_{j}: 0 \leq j \leq n\right),\left(P_{j}: 0 \leq j \leq n\right)$, and $\left(Y_{k}: k \in \mathbb{N}\right)$. Then, using (8), we have

$$
\begin{aligned}
\mathrm{E}\left(P_{n+1} Z_{n+1} \mid \mathcal{F}_{n}\right) & =\mathrm{E}\left(P_{n+1}\left[\sum_{j=1}^{Z_{n}} X_{j}+Y_{n}\right] \mid \mathcal{F}_{n}\right) \\
& =P_{n} \mathrm{E}\left(f_{n}^{\prime}(1)^{-1} \sum_{j=1}^{Z_{n}} X_{j} \mid \mathcal{F}_{n}\right)+P_{n} \mathrm{E}\left(f^{\prime}(1)^{-1}\right) Y_{n} \\
& =P_{n} \mathrm{E}\left(f_{n}^{\prime}(1)^{-1} Z_{n} \mathrm{E}\left(X_{1} \mid f_{n}\right) \mid \mathcal{F}_{n}\right)+P_{n} \mathrm{E}\left(f^{\prime}(1)^{-1}\right) Y_{n} \\
& =P_{n} Z_{n}+P_{n} \mathrm{E}\left(f^{\prime}(1)^{-1}\right) Y_{n} .
\end{aligned}
$$


So $P_{n} Z_{n}$ is a submartingale. Moreover,

$$
\mathrm{E}\left(P_{n} Z_{n} \mid \mathcal{F}_{0}\right)=Z_{0}+\sum_{j=0}^{n-1} \mathrm{E}\left(f^{\prime}(1)^{-1}\right)^{j+1} Y_{j} .
$$

By (10), if $\mathrm{E}\left(f^{\prime}(1)^{-1}\right)<1, P_{n} Z_{n}$ has bounded expectations and then converges a.s. to a finite random variable.

\section{Ergodicity and convergence for a random cell line}

Recall that $\left(Z_{n}\right)_{n \in \mathbb{N}}$ defined in the introduction is the number of parasites in a random cell line. The Markov chain $\left(Z_{n}\right)_{n \in \mathbb{N}}$ is a branching process in a random environment with statedependent immigration. The reproduction law is given by the PGF $f$, immigration in state 0 is distributed as $Y_{0}$, and immigration in state $k \geq 1$ is distributed as $Y_{1}$. More precisely, for every $n \in \mathbb{N}$, conditionally on $Z_{n}=x$,

$$
Z_{n+1}=Y_{x}^{(n)}+\sum_{j=1}^{x} X_{j}^{(n)}
$$

where

(i) $\left(X_{j}^{(n)}\right)_{j \in \mathbb{N}}$ and $Y_{x}^{(n)}$ are independent,

(ii) conditionally on $f_{n}=g$, the $\left(X_{j}^{(n)}\right)_{j \in \mathbb{N}}$ are i.i.d. with common PGF $g$,

(iii) for all $x \geq 1$ and $n \in \mathbb{N}, Y_{x}^{(n)} \stackrel{\mathrm{D}}{=} Y_{1}$.

We have the following results, which generalize those of the previous section to the case where immigration depends on whether the state is zero or not.

Theorem 3. (i) If $\mathrm{E}\left(\log \left(f^{\prime}(1)\right)\right)<0$ and $\max \left(\mathrm{E}\left(\log ^{+}\left(Y_{i}\right)\right): i=0,1\right)<\infty$, then there exists a finite random variable $Z_{\infty}$ such that, for every $k \in \mathbb{N}, Z_{n}$ starting from $k$ converges in distribution to $Z_{\infty}$ as $n \rightarrow \infty$.

Moreover, if there exists $q>0$ such that $\max \left(\mathrm{E}\left(Y_{i}^{q}\right): i=0,1\right)<\infty$, then, for every $\varepsilon>0$, there exist $0<r<1$ and $C>0$ such that, for all $n \in \mathbb{N}$ and $k \in \mathbb{N}$,

$$
\sum_{l=0}^{\infty}\left|\mathrm{P}_{k}\left(Z_{n}=l\right)-\mathrm{P}\left(Z_{\infty}=l\right)\right| \leq C k^{\varepsilon} r^{n} .
$$

(ii) If $\mathrm{E}\left(\log \left(f^{\prime}(1)\right)\right) \geq 0$ or $\max \left(\mathrm{E}\left(\log ^{+}\left(Y_{i}\right)\right): i=0,1\right)=\infty, Z_{n}$ converges in probability to $\infty$ as $n \rightarrow \infty$.

Note again that, by the Borel-Cantelli lemma, if $\mathrm{E}\left(\log ^{+}\left(Y_{1}\right)\right)=\infty$ then, for every $c>1$,

$$
\limsup _{n \rightarrow \infty} c^{-n} Z_{n}=\infty \quad \text { a.s., }
$$

since $Z_{n} \geq Y_{n}$ a.s.

The proof of Theorem 3(ii) in the critical or supercritical case $\left(\mathrm{E}\left(\log \left(f^{\prime}(1)\right)\right) \geq 0\right)$ is derived directly from Proposition 1, and so we now focus on the subcritical case:

$$
\mathrm{E}\left(\log \left(f^{\prime}(1)\right)\right)<0 .
$$


Recall that $T_{0}$ is the first time after 0 when $\left(Z_{n}\right)_{n \in \mathbb{N}}$ visits 0 . Using BPREI (see Section 4), we prove the following result in the subcritical case.

Lemma 2. If $\max \left(\mathrm{E}\left(\log ^{+}\left(Y_{i}\right)\right): i=0,1\right)<\infty$ then, for every $k \in \mathbb{N}, \mathrm{P}_{k}\left(T_{0}<\infty\right)=1$ and

$$
\sup _{n \in \mathbb{N}}\left\{\mathrm{P}_{k}\left(Z_{n} \geq l\right)\right\} \rightarrow 0 \text { as } l \rightarrow \infty
$$

Moreover, if there exists $q>0$ such that $\max \left(\mathrm{E}\left(Y_{i}^{q}\right): i=0,1\right)<\infty$, then, for every $\varepsilon>0$, there exist $r>0$ and $C>0$ such that, for all $n \in \mathbb{N}$ and $k \geq 1$,

$$
\mathrm{P}_{0}\left(T_{0} \geq n\right) \leq C r^{n}, \quad \mathrm{P}_{k}\left(T_{0} \geq n\right) \leq C k^{\varepsilon} r^{n} .
$$

Proof. We couple $\left(Z_{n}\right)_{n \in \mathbb{N}}$ with BPREI, $\left(\tilde{Z}_{n}\right)_{n \in \mathbb{N}}$, whose reproduction law is given by the random PGF $f$ (such as $\left(Z_{n}\right)_{n \in \mathbb{N}}$ ) and whose immigration $Y$ is defined by

$$
Y:=\max \left(Y_{0}, Y_{1}, \tilde{Y}\right),
$$

where $Y_{0}, Y_{1}$, and $\tilde{Y}$ are independent, and $\tilde{Y}$ is defined by

$$
\mathrm{P}(\tilde{Y}=0)=\frac{1}{2}, \quad \mathrm{P}(\tilde{Y}=n)=\alpha n^{-1-\varepsilon} \quad \text { for all } n \in \mathbb{N}^{*}, \quad \alpha:=\left[2 \sum_{j=1}^{\infty} j^{-1-\varepsilon}\right]^{-1}
$$

Thus, immigration $Y$ for $\tilde{Z}_{n}$ is stochastically larger than the immigration for $Z_{n}$ (whereas the reproduction law is the same), so that coupling gives

$$
Z_{n} \leq \tilde{Z}_{n} \quad \text { a.s. for all } n \in \mathbb{N} \text {. }
$$

Moreover, $\tilde{Z}_{n}$ is still subcritical. Recalling that $\min \left(\mathrm{P}\left(Y_{i}=0\right): i=0,1\right)>0, \mathrm{P}(\tilde{Y}=0)=\frac{1}{2}$, and that the expectation of the logarithm of every random variable is finite, we have

$$
\mathrm{E}\left(\log ^{+}(Y)\right)<\infty, \quad \mathrm{P}(Y=0)>0 .
$$

Then Proposition 1(i) ensures that $\tilde{Z}_{n}$ converges in distribution to a finite random variable, so that

$$
\sup _{n \in \mathbb{N}}\left\{\mathrm{P}_{k}\left(Z_{n} \geq l\right)\right\} \leq \sup _{n \in \mathbb{N}}\left\{\mathrm{P}_{k}\left(\tilde{Z}_{n} \geq l\right)\right\} \rightarrow 0 \quad \text { as } l \rightarrow \infty .
$$

Proposition 1(i) also ensures that, for every $k \in \mathbb{N}, \lim _{n \rightarrow \infty} \mathrm{P}_{k}\left(Z_{n}=0\right)>0$. Thus, for every $k \in \mathbb{N}, \mathrm{P}_{k}\left(\tilde{T}_{0}<\infty\right)=1$, and then $\mathrm{P}_{k}\left(T_{0}<\infty\right)=1$. This completes the first part of the lemma.

We now assume that there exists $q>0$ such that $\max \left(\mathrm{E}\left(Y_{i}^{q}\right): i=0,1\right)<\infty$. Moreover, $\mathrm{E}\left(\tilde{Y}^{\varepsilon / 2}\right)<\infty$, so letting $q^{\prime}=\min (\varepsilon / 2, q)$, we have

$$
\mathrm{E}\left(Y^{q^{\prime}}\right)<\infty .
$$

We can then apply Proposition 1(ii) to BPREI, $\left(\tilde{Z}_{n}\right)_{n \in \mathbb{N}}$, so that there exist $c, d>0$ such that, for every $n \in \mathbb{N}$,

$$
\mathrm{P}_{0}\left(\tilde{T}_{0}>n\right) \leq c \mathrm{e}^{-d n} .
$$

Recalling that, for all $k, n \in \mathbb{N}$,

$$
\mathrm{P}_{k}\left(T_{0} \geq n\right) \leq \mathrm{P}_{k}\left(\tilde{T}_{0} \geq n\right),
$$


we obtain $\mathrm{P}_{0}\left(T_{0} \geq n\right) \leq c \mathrm{e}^{-d n}$. Moreover, for every $k \in \mathbb{N}$,

$$
\mathrm{P}_{0}\left(\tilde{T}_{0}>n\right) \geq \mathrm{P}(Y \geq k) \mathrm{P}_{k}\left(\tilde{T}_{0} \geq n\right) .
$$

By the definition of $Y$, there exists $\beta>0$ such that, for every $n \in \mathbb{N}$,

$$
\mathrm{P}(Y \geq n) \geq \beta n^{-\varepsilon} .
$$

Using these inequalities gives

$$
\mathrm{P}_{k}\left(T_{0} \geq n\right) \leq \mathrm{P}_{k}\left(\tilde{T}_{0} \geq n\right) \leq \beta^{-1} k^{\varepsilon} \mathrm{P}_{0}\left(\tilde{T}_{0}>n\right) \leq \beta^{-1} c k^{\varepsilon} \mathrm{e}^{-d n} .
$$

This completes the proof.

Proof of Theorem 3(i) and (ii) in the subcritical case: $\mathrm{E}\left(\log \left(f^{\prime}(1)\right)\right)<0$. Split the proof into four cases.

Case 1. $\max \left(\mathrm{E}\left(\log ^{+}\left(Y_{i}\right)\right): i=0,1\right)<\infty$.

Case 2. There exists $q>0$ such that $\max \left(\mathrm{E}\left(Y_{i}^{q}\right): i=0,1\right)<\infty$.

Case 3. $\mathrm{E}\left(\log ^{+}\left(Y_{1}\right)\right)=\infty$.

Case 4. $\mathrm{E}\left(\log ^{+}\left(Y_{0}\right)\right)=\infty$.

First, note that $\mathrm{P}\left(Y_{0}=0\right)>0$ ensures that $\mathrm{P}_{0}\left(Z_{1}=0\right)>0$, and we can use the results of Section 3.2.

Case 1. In this case, by Lemma $2,\left(Z_{n}\right)_{n \in \mathbb{N}}$ is bounded in distribution:

$$
\sup _{n \in \mathbb{N}}\left\{\mathrm{P}_{0}\left(Z_{n} \geq l\right)\right\} \rightarrow 0 \quad \text { as } l \rightarrow \infty .
$$

If $\mathrm{E}_{0}\left(T_{0}\right)=\infty$ then $Z_{n} \rightarrow \infty$ in $\mathrm{P}_{0}$ by Lemma 1(ii), which is in contradiction with the previous limit.

Then $\mathrm{E}_{0}\left(T_{0}\right)<\infty$. We now prove that, for all $k \geq 1, \mathrm{E}_{k}\left(T_{0}\right)<\infty$ by a coupling argument. Let $k \geq 1$, and change only the immigration to obtain a Markov process, $\left(\tilde{Z}_{n}\right)_{n \in \mathbb{N}}$, which is larger than $\left(Z_{n}\right)_{n \in \mathbb{N}}$ :

$$
\tilde{Z}_{n} \geq Z_{n} \quad \text { a.s. for all } n \in \mathbb{N} \text {. }
$$

Its immigrations, $\tilde{Y}_{0}$ and $\tilde{Y}_{1}$, satisfy

$$
\begin{aligned}
& \tilde{Y}_{1} \stackrel{\mathrm{D}}{=} Y_{1}, \quad \mathrm{P}\left(\tilde{Y}_{0} \geq n\right) \geq \mathrm{P}\left(Y_{0} \geq n\right) \quad \text { for all } n \in \mathbb{N}, \\
& \mathrm{P}\left(\tilde{Y}_{0} \geq k\right)>0, \quad \max \left(\mathrm{E}\left(\log \left(\tilde{Y}_{i}\right)\right): i=0,1\right)<\infty .
\end{aligned}
$$

Then, we again have $\mathrm{E}_{0}\left(\tilde{T}_{0}\right)<\infty$, which entails that $\mathrm{E}_{k}\left(\tilde{T}_{0}\right)<\infty$ since $\mathrm{P}\left(\tilde{Y}_{0} \geq k\right)>0$. As, for every $n \in \mathbb{N}, \tilde{Z}_{n} \geq Z_{n}$ a.s., we have

$$
\mathrm{E}_{k}\left(T_{0}\right) \leq \mathrm{E}_{k}\left(\tilde{T}_{0}\right)<\infty
$$

Then Lemma 1(i) ensures that, for every $k \in \mathbb{N},\left(Z_{n}\right)_{n \in \mathbb{N}}$ converges in distribution to a finite random variable $Z_{\infty}$, which does not depend on $k$ and verifies $\mathrm{P}\left(Z_{\infty}=0\right)>0$. 
Case 2. By Lemma 1(i) we have

$$
\begin{aligned}
& \sum_{l \in \mathbb{N}}\left|\mathrm{P}_{k}\left(Z_{n}=l\right)-\mathrm{P}\left(Z_{\infty}=l\right)\right| \\
& \quad \leq A\left[\sup _{n / 2 \leq l \leq n}\left\{\left|u_{l}-u_{\infty}\right|\right\}+\mathrm{E}_{0}\left(T_{0} \mathbf{1}_{\left\{T_{0}>n / 4\right\}}\right)+\mathrm{E}_{k}\left(T_{0} \mathbf{1}_{\left\{T_{0}>n / 4\right\}}\right)\right] .
\end{aligned}
$$

Moreover, by Lemma 2, for every $\varepsilon>0$, there exists $C>0$ such that

$$
\mathrm{P}_{k}\left(T_{0} \geq n\right) \leq C k^{\varepsilon} r^{n}, \quad \mathrm{P}_{0}\left(T_{0} \geq n\right) \leq C r^{n} .
$$

So, for every $r^{\prime} \in(r, 1), \mathrm{E}_{0}\left(\exp \left(-\log (r) T_{0}\right)\right)<\infty$. Then, by Kendall's renewal theorem [14], there exist $\rho \in(0,1)$ and $c>0$ such that, for every $n \in \mathbb{N}$,

$$
\left|u_{n}-u_{\infty}\right| \leq c \rho^{n}
$$

Finally, (12) ensures that there exists $D>0$ such that, for every $n \in \mathbb{N}$,

$$
\begin{aligned}
& \mathrm{E}_{0}\left(T_{0} \mathbf{1}_{\left\{T_{0}>n / 4\right\}}\right) \leq D n r^{n / 4}, \\
& \mathrm{E}_{k}\left(T_{0} \mathbf{1}_{\left\{T_{0}>n / 4\right\}}\right) \leq D n k^{\varepsilon} r^{n / 4} .
\end{aligned}
$$

Combining these two inequalities with (11) and (13), we obtain

$$
\sum_{l \in \mathbb{N}}\left|\mathrm{P}_{k}\left(Z_{n}=l\right)-\mathrm{P}\left(Z_{\infty}=l\right)\right| \leq A\left[c \rho^{n}+D n r^{n / 4}+D n k^{\varepsilon} r^{n / 4}\right],
$$

which completes the proof of Case 2 .

Case 3. Change the immigration of $\left(Z_{n}\right)_{n \in \mathbb{N}}$ to obtain BPREI, $\left(\tilde{Z}_{n}\right)_{n \in \mathbb{N}}$, whose immigration is distributed as $Y_{1}$ and whose reproduction law is still given by $f$. Then Proposition 1(i) and $\mathrm{E}\left(\log ^{+}\left(Y_{1}\right)\right)=\infty$ ensure that $\left(\tilde{Z}_{n}\right)_{n \in \mathbb{N}}$ starting from 0 tends in distribution to $\infty$.

Then Lemma 1(i) entails that $\mathrm{E}_{0}\left(\tilde{T}_{0}\right)=\infty$, so that, for every $k \geq 1$,

$$
\mathrm{E}_{k}\left(\tilde{T}_{0}\right) \geq \mathrm{E}_{0}\left(\tilde{T}_{0}\right)=\infty
$$

since the BPREI, $\left(\tilde{Z}_{n}\right)_{n \in \mathbb{N}}$, starting from $k \geq 1$ are stochastically larger than $\left(\tilde{Z}_{n}\right)_{n \in \mathbb{N}}$ starting from 0 .

Moreover, under $\mathrm{P}_{k},\left(Z_{n}\right)_{n \in \mathbb{N}}$ is equal to $\left(\tilde{Z}_{n}\right)_{n \in \mathbb{N}}$ until time $T_{0}=\tilde{T}_{0}$. So $\mathrm{E}_{k}\left(T_{0}\right)=\infty$. Let $k \geq 1$ such that $\mathrm{P}_{0}\left(Z_{1}=k\right)>0$. Then $\mathrm{E}_{0}\left(T_{0}\right) \geq \mathrm{P}_{0}\left(Z_{1}=k\right) \mathrm{E}_{k}\left(T_{0}-1\right)$. This entails that

$$
\mathrm{E}_{0}\left(T_{0}\right)=\infty
$$

By Lemma 1(ii), $\left(Z_{n}\right)_{n \in \mathbb{N}}$ starting from any $k \in \mathbb{N}$ tends to $\infty$ in probability.

Case 4. Denote by

$$
X_{j}:=\mathrm{P}\left(Z_{j}>0 \mid Z_{j-1}=1, f_{j-1}\right), \quad j \geq 1,
$$

the survival probability in environment $f_{j-1}$ and introduce the following random walk:

$$
S_{n}=\sum_{j=1}^{n} \log \left(X_{j}\right)
$$


Then

$$
\mathrm{P}_{1}\left(Z_{n}>0 \mid\left(f_{0}, f_{1}, \ldots, f_{n-1}\right)\right) \geq \prod_{j=1}^{n} X_{j}=\exp \left(S_{n}\right) \quad \text { a.s. }
$$

so that

$$
\begin{aligned}
\mathrm{P}_{k}\left(Z_{n}>0 \mid\left(f_{0}, f_{1}, \ldots, f_{n-1}\right)\right) & =1-\mathrm{P}_{k}\left(Z_{n}=0 \mid\left(f_{0}, f_{1}, \ldots, f_{n-1}\right)\right) \\
& =1-\left[1-\mathrm{P}_{1}\left(Z_{n}>0 \mid\left(f_{0}, f_{1}, \ldots, f_{n-1}\right)\right)\right]^{k} \\
& \geq 1-\left[1-\exp \left(S_{n}\right)\right]^{k} \text { a.s. }
\end{aligned}
$$

Thus,

$$
\mathrm{P}_{k}\left(Z_{n}>0\right) \geq \mathrm{E}\left(1-\left[1-\exp \left(S_{n}\right)\right]^{k}\right) .
$$

Using the Markov property, we have

$$
\begin{aligned}
\mathrm{E}_{0}\left(T_{0}+1\right) & \geq \sum_{k=1}^{\infty} \mathrm{P}\left(Y_{0}=k\right) \mathrm{E}_{k}\left(T_{0}\right) \\
& =\sum_{k=1}^{\infty} \mathrm{P}\left(Y_{0}=k\right) \sum_{n=1}^{\infty} \mathrm{P}_{k}\left(T_{0} \geq n\right) \\
& \geq \sum_{k=1}^{\infty} \mathrm{P}\left(Y_{0}=k\right) \sum_{n=1}^{\infty} \mathrm{P}_{k}\left(Z_{n}>0\right) \\
& \geq \sum_{k=1}^{\infty} \mathrm{P}\left(Y_{0}=k\right) \sum_{n=1}^{\infty} \mathrm{E}\left(1-\left[1-\exp \left(S_{n}\right)\right]^{k}\right)
\end{aligned}
$$

Moreover, for all $x \in[0,1)$ and $k \geq 0, \exp (k \log (1-x)) \leq \exp (-k x)$, and by the law of large numbers, $S_{n} / n$ tends a.s. to $\mathrm{E}\left(X_{1}\right)<0$ so that there exists $n_{0} \geq 1$ such that, for every $n \geq n_{0}$,

$$
\mathrm{P}\left(\frac{S_{n}}{n} \geq \frac{3 \mathrm{E}\left(X_{1}\right)}{2}\right) \geq \frac{1}{2} .
$$

We then obtain

$$
\begin{aligned}
\mathrm{E}_{0}\left(T_{0}+1\right) & \geq \sum_{k=1}^{\infty} \mathrm{P}\left(Y_{0}=k\right) \sum_{n=1}^{\infty} \mathrm{E}\left(1-\exp \left(-k \exp \left(S_{n}\right)\right)\right) \\
& \geq\left[1-\mathrm{e}^{-1}\right] \sum_{n=1}^{\infty} \sum_{k=1}^{\infty} \mathrm{P}\left(k \exp \left(S_{n}\right) \geq 1\right) \mathrm{P}\left(Y_{0}=k\right) \\
& \geq\left[1-\mathrm{e}^{-1}\right] \sum_{n=n_{0}}^{\infty} \mathrm{P}\left(\frac{S_{n}}{n} \geq \frac{3 \mathrm{E}\left(X_{1}\right)}{2}\right) \sum_{k \geq \exp \left(-3 n \mathrm{E}\left(X_{1}\right) / 2\right)}^{\infty} \mathrm{P}\left(Y_{0}=k\right) \\
& \geq 2^{-1}\left[1-\mathrm{e}^{-1}\right] \sum_{n=n_{0}}^{\infty} \mathrm{P}\left(Y_{0} \geq \exp \left(-\frac{3 n \mathrm{E}\left(X_{1}\right)}{2}\right)\right) \\
& \geq 2^{-1}\left[1-\mathrm{e}^{-1}\right] \sum_{n=n_{0}}^{\infty} \mathrm{P}\left(\beta \log \left(Y_{0}\right) \geq n\right),
\end{aligned}
$$


where $\beta:=\left[-3 \mathrm{E}\left(X_{1}\right) / 2\right]^{-1}>0$. Then $\mathrm{E}\left(\log \left(Y_{0}\right)\right)=\infty$ ensures that $\mathrm{E}_{0}\left(T_{0}+1\right)=\infty$, so

$$
\mathrm{E}_{0}\left(T_{0}\right)=\infty
$$

We conclude that $\left(Z_{n}\right)_{n \in \mathbb{N}}$ tends to $\infty$ in $\mathrm{P}_{k}$ using Lemma 1(ii).

\section{Asympotics for proportions of cells with a given number of parasites}

\subsection{Asymptotics without contamination}

Here we consider the case where there is no contamination, i.e. $Y_{0}=Y_{1}=0$ a.s., and we determine when the organism recovers, that is, when the number of contaminated cells becomes negligible compared to the total number of cells. We obtain the same result as Theorem 1 of [8] for the more general model considered here. Denote by $N_{n}$ the number of contaminated cells.

Proposition 2. As $n$ increases, $N_{n} / 2^{n}$ decreases. If $\mathrm{E}\left(\log \left(f^{\prime}(1)\right)\right) \leq 0$ then $N_{n} / 2^{n} \rightarrow 0$ a.s. as $n \rightarrow \infty$. Otherwise, $N_{n} / 2^{n} \rightarrow 0$ as $n \rightarrow \infty$ if and only if all parasites die out, which happens with a probability of less than 1 .

Example. Consider the case of the random binomial repartition of parasites mentioned in the introduction. Let $Z \in \mathbb{N}$ be a random variable, and let $\left(P_{i}\right)_{i \in \mathbb{T}}$ be an i.i.d. sequence distributed as a random variable $P \in[0,1]$, such that $P \stackrel{\mathrm{D}}{=} 1-P$. In every generation, each parasite gives birth independently to a random number of parasites distributed as $Z$. When cell $i$ divides, conditionally on $P_{i}=p$, each parasite of cell $i$ goes independently into the first daughter cell with probability $p$ (or it goes into the second daughter cell with probability $1-p$ ). Then,

$$
\mathrm{P}\left(f^{\prime}(1) \in \mathrm{d} x\right)=\mathrm{P}(\mathrm{E}(Z) P \in \mathrm{d} x) .
$$

Thus, the organism recovers a.s. (i.e. $N_{n} / 2^{n}$ tends a.s. to 0 ) if and only if

$$
\log (\mathrm{E}(Z)) \leq \mathrm{E}\left(\log \left(\frac{1}{P}\right)\right) .
$$

The same criterion also holds for the case in which the offspring of each parasite goes a.s. into the same daughter cell (here, $p$ is the probability that this offspring goes into the first daughter cell.)

Proof of Proposition 2. Note that $N_{n} / 2^{n}$ decreases to $L$ as $n \rightarrow \infty$, since one infected cell has at most two daughter cells which are infected. Moreover, for every $n \in \mathbb{N}$,

$$
\begin{aligned}
\mathrm{E}\left(\frac{N_{n}}{2^{n}}\right) & =\frac{\mathrm{E}\left(\sum_{i \in \mathbb{G}_{n}} \mathbf{1}_{\left\{Z_{i}>0\right\}}\right)}{2^{n}} \\
& =\sum_{i \in \mathbb{G}_{n}} \frac{1}{2^{n}} \mathrm{E}\left(\mathbf{1}_{\left\{Z_{i}>0\right\}}\right) \\
& =\sum_{i \in \mathbb{G}_{n}} \mathrm{P}\left(\left(a_{0}, \ldots, a_{n-1}\right)=i\right) \mathrm{P}\left(Z_{i}>0\right) \\
& =\mathrm{P}\left(Z_{n}>0\right)
\end{aligned}
$$

If $\mathrm{E}\left(\log \left(f^{\prime}(1)\right)\right) \leq 0$ (subcritical or critical case) then $\mathrm{P}\left(Z_{n}>0\right)$ tends to 0 as $n \rightarrow \infty$ (see Section 3.1). Thus, $\mathrm{E}(L)=0$ and $N_{n} / 2^{n}$ tends to 0 a.s. as $n \rightarrow \infty$.

If $\mathrm{E}\left(\log \left(f^{\prime}(1)\right)\right)>0$ (supercritical case) then $\mathrm{P}\left(Z_{n}>0\right)$ tends to a positive value, which is equal to $\mathrm{P}(L>0)>0$. We complete the proof with Lemma 3, below. 
Let us prove the following zero-one law, where $P_{n}$ is the total number of parasites in generation $n$.

Lemma 3. If $\mathrm{E}\left(\log \left(f^{\prime}(1)\right)\right)>0$ then

$$
\left\{\lim _{n \rightarrow \infty} \frac{N_{n}}{2^{n}}>0\right\}=\left\{\text { for all } n \in \mathbb{N}: P_{n}>0\right\} \text { a.s. }
$$

Proof. First, we prove that, conditionally on the nonextinction of parasites, for every $K \in \mathbb{N}$, there exists a.s. a generation $n$ such that $N_{n} \geq K$. Letting $K \in \mathbb{N}$, we fix $p$ as the first integer such that $2^{p} \geq K$. Then $q:=\mathrm{P}_{1}\left(N_{p} \geq K\right)>0$ since $\mathrm{P}\left(N_{1}=2\right)>0$.

Either the number of infected cells in generation $p$ is more than $K$, which happens with probability $q$, or we can choose in generation $p$ an infected cell, which we denote by $i(1)$, since parasites have not died out. Then, with probability larger than $q$, the number of infected cells in generation $p$ of the subtree rooted in cell $i$ (1) contains more than $K$ parasites. Note that this probability is exactly equal to $q$ if and only if the infected cell $i$ (1) contains one single parasite. Recursively, we find a.s. a generation $n$ such than $N_{n} \geq K$.

Then, recalling that we still work conditionally on the nonextinction of parasites, the stopping time $T:=\inf \left\{n \in \mathbb{N}: N_{n} \geq K\right\}<\infty$ a.s. We now also condition by $T=n$ and $N_{T}=k$. We can then choose one parasite in every infected cell in generation $n$, which we label by $1 \leq j \leq k$, and we denote by $N_{p}^{(j)}$ the number of cells in generation $n+p$ infected by parasites whose ancestor in generation $n$ is the parasite $j$. By the branching property, the integers $\left(N_{p}^{(j)}: 1 \leq j \leq k\right)$ are i.i.d. and $N_{p}^{(j)} / 2^{p} \rightarrow L^{(j)}$ as $p \rightarrow \infty$, where the $\left(L^{(j)}: 1 \leq j \leq k\right)$ are independent and $\mathrm{P}\left(L^{(j)}>0\right)=\mathrm{P}(L>0)>0$ for every $1 \leq j \leq k$. Using the fact that

$$
N_{n+p} \geq \sum_{j=1}^{k} N_{p}^{(j)} \quad \text { a.s. }
$$

and as $k \geq K$, we obtain

$$
\lim _{p \rightarrow \infty} \frac{N_{n+p}}{2^{p}} \geq \max \left(L^{(j)}: 1 \leq j \leq K\right) \quad \text { a.s. }
$$

$\operatorname{As} \sup \left(L^{(j)}: j \in \mathbb{N}\right)=\infty$ a.s., letting $K \rightarrow \infty$ ensures that, a.s., $N_{p} / 2^{p}$ does not tend to 0 .

\subsection{Asymptotics with contamination in the $\mathrm{E}\left(\log \left(f^{\prime}(1)\right)\right)<0$ and $\max \left(\mathrm{E}\left(\log ^{+}\left(Y_{i}\right)\right): i=\right.$ 0, 1) $<\infty$ cases}

Define $F_{k}(n)$ to be the proportion of cells with $k$ parasites in generation $n$ :

$$
F_{k}(n):=\frac{\#\left\{i \in \mathbb{G}_{n}: Z_{i}=k\right\}}{2^{n}}, \quad k \in \mathbb{N} .
$$

We introduce the Banach space, $l^{1}(\mathbb{N})$, and the subset of frequencies, $\mathbb{S}^{1}(\mathbb{N})$, which we endow with the norm $\|\cdot\|_{1}$ defined by

$$
\begin{gathered}
l^{1}(\mathbb{N}):=\left\{\left(x_{j}\right)_{j \in \mathbb{N}}: \sum_{j=0}^{\infty}\left|x_{j}\right|<\infty\right\}, \quad\left\|\left(x_{j}\right)_{j \in \mathbb{N}}\right\|_{1}=\sum_{j=0}^{\infty}\left|x_{j}\right|, \\
\mathbb{S}^{1}(\mathbb{N}):=\left\{\left(f_{j}\right)_{j \in \mathbb{N}}: \text { for all } j \in \mathbb{N}, f_{j} \in \mathbb{R}^{+}, \sum_{j=0}^{\infty} f_{j}=1\right\} .
\end{gathered}
$$


The main argument used here is the law of large numbers proved by Guyon [12] for asymmetric Markov chains indexed by a tree.

Theorem 4. If $\mathrm{E}\left(\log \left(f^{\prime}(1)\right)\right)<0$ and $\max \left(\mathrm{E}\left(\log ^{+}\left(Y_{i}\right)\right): i=0,1\right)<\infty$, then $\left(F_{k}(n)\right)_{k \in \mathbb{N}}$ converges in probability in $\mathbb{S}^{1}(\mathbb{N})$ to a deterministic sequence $\left(f_{k}\right)_{k \in \mathbb{N}}$ as $n \rightarrow \infty$, such that $f_{0}>0$ and $\sum_{k=0}^{\infty} f_{k}=1$. Moreover, for every $k \in \mathbb{N}, f_{k}=\mathrm{P}\left(Z_{\infty}=k\right)$.

Proof. Recall that $\left(Z_{i}\right)_{i \in \mathbb{T}}$ is a Markov chain indexed by a tree and that we are in the framework of bifurcating Markov chains studied in [12]. Thanks to the ergodicity of the number of parasites in a random cell line proved in the previous section (Theorem 1(i)), we can directly apply Theorem 8 of [12] to obtain the convergence of proportions of cells with a given number of parasites.

It seems that we cannot apply Theorem 14 or Corollary 15 of [12] to obtain almost-sure convergence of proportions, because of the $k^{\varepsilon}$ term in the estimation of Theorem 1. For examples, we refer the reader to Section 6.1.

Again, using [12], we can also prove a law of large numbers and a central limit theorem for the proportions of cells with a given number of parasites before generation $n$. Define, for every $n \in \mathbb{N}$,

$$
P_{k}(n):=\frac{\#\left\{i \in \bigcup_{0 \leq i \leq n} \mathbb{G}_{i}: Z_{i}=k\right\}}{2^{n+1}}, \quad k \in \mathbb{N} .
$$

Theorem 5. If $\mathrm{E}\left(\log \left(f^{\prime}(1)\right)\right)<0$ and $\max \left(\mathrm{E}\left(\log ^{+}\left(Y_{i}\right)\right): i=0,1\right)<\infty$, then $\left(P_{k}(n)\right)_{k \in \mathbb{N}}$ converges in probability in $\mathbb{S}^{1}(\mathbb{N})$ to the deterministic sequence $\left(f_{k}\right)_{k \in \mathbb{N}}$ as $n \rightarrow \infty$. Moreover, for every $k \in \mathbb{N}, \sqrt{n}\left(P_{k}(n)-f_{k}\right)$ converges in distribution to a centered normal law as $n \rightarrow \infty$, with a nonexplicit variance.

Proof. Use Theorem 1(i) again and Theorem 8 of [12] to prove the law of large numbers. For the central limit theorem, use Theorem 19 of [12] by letting $F$ be the set of continuous functions taking values in $[0,1]$.

6.3. Asymptotics with contamination in the $\mathrm{E}\left(\log \left(f^{\prime}(1)\right)\right) \geq 0$ or $\max \left(\mathrm{E}\left(\log ^{+}\left(Y_{i}\right)\right): i=\right.$ $0,1)=\infty$ case

In this case, cells become infinitely infected as the number of generations tends to $\infty$.

Theorem 6. If $\mathrm{E}\left(\log \left(f^{\prime}(1)\right)\right) \geq 0$ or $\max \left(\mathrm{E}\left(\log ^{+}\left(Y_{i}\right)\right): i=0,1\right)=\infty$, then, for every $k \in \mathbb{N}$, $F_{k}(n)$ tends to 0 as $n \rightarrow \infty$. That is, for every $K \in \mathbb{N}$,

$$
\lim _{n \rightarrow \infty} \frac{\#\left\{i \in \mathbb{G}_{n}: Z_{i} \geq K\right\}}{2^{n}} \stackrel{\mathrm{P}}{=} 1 \text {. }
$$

Proof. By Fubini's theorem we have

$$
\begin{aligned}
\mathrm{E}\left(\frac{\#\left\{i \in \mathbb{G}_{n}: Z_{i} \geq K\right\}}{2^{n}}\right) & =\frac{\sum_{i \in \mathbb{G}_{n}} \mathrm{P}\left(Z_{i} \geq K\right)}{2^{n}} \\
& =\sum_{i \in \mathbb{G}_{n}} \mathrm{P}\left(\left(a_{0}, \ldots, a_{n-1}\right)=i\right) \mathrm{P}\left(Z_{i} \geq K\right) \\
& =\mathrm{P}\left(Z_{n} \geq K\right) .
\end{aligned}
$$

By Theorem $1, \mathrm{P}\left(Z_{n} \geq K\right)$ tends to 1 , so $1-\#\left\{i \in \mathbb{G}_{n}: Z_{i} \geq K\right\} / 2^{n}$ converges to 0 in $L^{1}$, which gives the result. 


\section{Asymptotics for the number of parasites}

We assume here that parasites multiply following a Galton-Watson process with deterministic mean $m$, independently of the cell they belong to. That is, $s \mapsto \hat{f}(s, s)$ is deterministic and every parasite multiplies independently with reproduction law whose PGF is equal to $g: s \mapsto \hat{f}(s, s)$. Moreover, we assume that contamination of a cell does not depend on the number of enclosed parasites. That is,

$$
Y \stackrel{\mathrm{D}}{=} Y_{0} \stackrel{\mathrm{D}}{=} Y_{1} .
$$

Let $P_{n}$ be the number of parasites in generation $n$. Without contamination, in the supercritical case, $m>1$, it is well known that either $P_{n}$ becomes extinct or $P_{n} / m^{n}$ converges to a positive finite random variable. In the presence of contamination, we have the following result.

Proposition 3. If $\mathrm{E}(Y)<\infty$ and $\mathrm{P}\left(Y_{0}>0\right)>0, \log \left(P_{n}\right) / n$ converges in $\mathrm{P}$ to $\log (\max (2, m))$. than

Proof. First, we prove the lower bound. This is a consequence of the fact that $P_{n}$ is larger

(i) the total number of parasites $P_{n}^{1}$ which contaminate cells of generation $n$,

(ii) the number of parasites $P_{n}(p)$ in generation $n$ with the same given parasite ancestor in generation $p$.

Indeed, $P_{n}^{1}$ is the sum of $2^{n}$ i.i.d. random variables with mean $\mathrm{E}(Y)$, so the law of large numbers ensures that

$$
\frac{P_{n}^{1}}{2^{n}} \rightarrow \mathrm{E}(Y)>0 \quad \text { in } \mathrm{P} \text {, as } n \rightarrow \infty .
$$

Then, since $P_{n} \geq P_{n}^{1}$ a.s. for every $n \in \mathbb{N}$,

$$
P_{n} \rightarrow \infty \text { in } \mathrm{P} \text {, as } n \rightarrow \infty \text {. }
$$

Moreover, for every $p<n$,

$$
\frac{P_{n}(p)}{m^{n-p}} \rightarrow W \quad \text { a.s., as } n \rightarrow \infty,
$$

with $\mathrm{P}(W>0)>0$. Now let $P_{n}^{2}$ be the sum of the number of descendants in generation $n$ of each parasite of generation $p$. We then obtain the sum of $P_{p}$ i.i.d. quantities distributed as $P_{n}(p)$. Then (14) and (15) ensure that we can choose $p$ such that

$$
\frac{P_{n}^{2}}{m^{n-p}} \rightarrow W^{\prime} \quad \text { a.s., as } n \rightarrow \infty,
$$

with $\mathrm{P}\left(W^{\prime}>0\right) \geq 1-\varepsilon$.

Using the fact that $N_{n}$ is larger than $P_{n}^{1}$ and $P_{n}^{2}$ ensures that, for every $\varepsilon>0$,

$$
\limsup _{n \rightarrow \infty} \mathrm{P}\left(\frac{\log \left(P_{n}\right)}{n} \leq \log (\max (2, m))\right)<\varepsilon .
$$

Letting $\varepsilon \rightarrow 0$ gives the lower bound. 
We now prove the upper bound. Note that the total number of parasites in generation $n$ can be written as

$$
P_{n}=\sum_{i=1}^{n} \sum_{j=1}^{2^{i}} \sum_{k=1}^{Y^{i, j}} Z_{k}^{i, j},
$$

where $Y^{i, j}$ is the number of parasites which contaminate the $j$ th cell of generation $i$, labeling these parasites as $1 \leq k \leq Y^{i, j}, Z_{k}^{i, j}$ is the number of descendants in generation $n$ of the $k$ th parasites. Moreover, $\left(Y^{i, j}: i \in \mathbb{N}, j \in \mathbb{N}\right)$ are identically distributed and independent of $\left(Z_{p}^{i, j}(k), i \in \mathbb{N}, j \in \mathbb{N}, k \in \mathbb{N}\right),\left(Z_{k}^{i, j}, i \in \mathbb{N}, j \in \mathbb{N}, k \in \mathbb{N}\right)$ are independent, and $Z_{p}^{i, j}(k)$ is the population of a Galton-Watson process in generation $n-i$ with offspring PGF equal to $g$. Thus,

$$
\begin{aligned}
\mathrm{E}\left(P_{n}\right) & =\sum_{i=1}^{n} \sum_{j=1}^{2^{i}} \mathrm{E}\left(\sum_{k=1}^{Y^{i, j}} Z_{k}^{i, j}\right) \\
& =\sum_{i=1}^{n} \sum_{j=1}^{2^{i}} \mathrm{E}\left(Y^{i, j}\right) \mathrm{E}\left(Z_{k}^{i, j}\right) \\
& =\mathrm{E}(Y) \sum_{i=1}^{n} \sum_{j=1}^{2^{i}} m^{n-i} \\
& =2 \mathrm{E}(Y) \frac{m^{n}-2^{n}}{m-2} \quad \text { if } m \neq 2 .
\end{aligned}
$$

If $m=2$ then $\mathrm{E}\left(P_{n}\right)=\mathrm{E}(Y) n m^{n}$. This gives the upper bound by the Markov inequality and completes the proof.

\section{References}

[1] Asmussen, S. and Hering, H. (1983). Branching Processes (Progress Prob. Statist. 3), Birkhäuser, Boston, MA.

[2] Athreya, K. B. And Karlin, S. (1971). Branching processes with random environments. II. Limit theorems. Ann. Math. Statist. 42, 1843-1858.

[3] Athreya, K. B. and Karlin, S. (1971). On branching processes with random environments. I. Extinction probabilities. Ann. Math. Statist. 42, 1499-1520.

[4] Athreya, K. B. And Ney, P. E. (2004). Branching Processes. Dover Publications, Mineola, NY.

[5] Athreya, K. B. ANd KANG, H.-J. (1998). Some limit theorems for positive recurrent branching Markov chains. I. Adv. Appl. Prob. 30, 693-710.

[6] Athreya, K. and Kang, H.-J. (1998). Some limit theorems for positive recurrent branching Markov chains. II. Adv. Appl. Prob. 30, 711-722.

[7] Afanasyev, V. I., Geiger, J., Kersting, G. and Vatutin, V. A. (2005). Criticality for branching processes in a random environment. Ann. Prob. 33, 645-673.

[8] Bansaye, V. (2008). Proliferating parasites in dividing cells: Kimmel's branching model revisited. Ann. Appl. Prob. 18, 967-996.

[9] Benjamini, I. and Peres, Y. (1994). Markov chains indexed by trees. Ann. Prob. 22, 219-243.

[10] Feller, W. (1966). An Introduction to Probability Theory and Its Applications, Vol. II. John Wiley, New York.

[11] Geiger, J., Kersting, G. And Vatutin, V. A. (2003). Limit theorems for subcritical branching processes in a random environment. Ann. Inst. H. Poincaré Prob. Statist. 39, 593-620.

[12] GUYON, J. (2007). Limit theorems for bifurcating Markov chains. Application to the detection of cellular aging. Ann. Appl. Prob. 17, 1538-1569.

[13] KEY, E. S. (1987). Limiting distributions and regeneration times for multitype branching processes with immigration in a random environment. Ann. Prob. 15, 344-353. 
[14] Kendall, D. G. (1959). Unitary dilations of Markov transition operators, and the corresponding integral representations for transition-probability matrices. In Probability and Statistics: The Harald Cramér Volume, John Wiley, New York, pp. 139-161.

[15] Kimmel, M. (1997). Quasistationarity in a branching model of division-within-division. In Classical and Modern Branching Processes (Minneapolis, MN, 1994; IMA Vol. Math. Appl. 84), Springer, New York, pp. 157-164.

[16] Kozlov, M. V. (1976). The asymptotic behavior of the probability of non-extinction of critical branching processes in a random environment. Theory Prob. Appl. 21, 813-825.

[17] Lyons, R. ANd Peres, Y. (2005). Probability on Trees and Networks. Available at http://mypage.iu.edu/ rdlyons/ prbtree/prbtree.html.

[18] Roitershtein, A. (2007). A note on multitype branching processes with immigration in a random environment. Ann. Prob. 35, 1573-1592.

[19] Smith, W. L. AND Wilkinson, W. E. (1969). On branching processes in random environments. Ann. Math. Statist. 40, 814-827.

[20] Stewart, E. J., Madden, R., Paul, G. and Taddei, F. (2005). Aging and death in an organism that reproduces by morphologically symmetric division. PLoS Biol. 3, e45. 